\title{
COMPARATIVE EVALUATION OF NATURAL AND SYNTHETIC SUPERDISINTEGRANTS FOR FAST DISSOLVING TABLET
}

\author{
*Patni Sonal D ${ }^{1}$, Gondkar S.B ${ }^{1}$, Darekar A.B ${ }^{1}$, Sharma Y.P ${ }^{2}$, Saudagar R.B ${ }^{1}$ \\ ${ }^{1}$ Department of Quality Assurance Techniques, R. G. Sapkal College of Pharmacy, Anjaneri, Nashik, M.S, India- 422213. \\ ${ }^{2}$ Department of Pharmaceutics, S.N.D. College of Pharmacy, Babhulgaon, Yeola, Nashik, M.S, India- 423401. \\ *Corresponding Author's Email: sonalpatani@yahoo.com Contact no.:9960662447
}

\begin{abstract}
In the present study, fast dissolving tablets of Salbutamol sulphate were prepared by direct compression method for better patient compliance and immediate action in asthma. The tablets were prepared by using synthetic superdisintegrants (Croscarmellose sodium and Sodium starch glycolate) and natural superdisintegrant (mucilage of Plantago ovata and Plantago ovata husk powder) at different concentrations as $2,4,6,8$ and $10 \%$. The Plantago ovata mucilage was extracted from the seeds of Plantago ovata (Plantaginaceae).The tablets were characterized for weight variation, hardness, friability, disintegration time, wetting time, water absorption ratio, drug content and in vitro dissolution tests. The Drug excipients compatibility study was performed by DSC and IR spectroscopy and no incompatibility was found. The tablets were subjected for accelerated stability study at $40^{\circ} \mathrm{C} 175 \% \mathrm{RH}$ and were found to be stable. The results clearly shows Natural superdisintegrants requires less disintegration time as compared to synthetic superdisintegrants. Hence present study reveals that the fast dissolving tablets prepared by using mucilage of Plantago ovata and husk powder of Plantago ovata as superdisintegrants having better appearance and rapid disintegration time.

Key-words: Fast dissolving tablets, Superdisintegrants, Plantago ovata, Croscarmellose sodium and Sodium starch glycolate.
\end{abstract}

\section{INTRODUCTION}

Fast dissolving tablets (FDT) are solid single-unit dosage forms that are placed in the mouth, allowed to disperse/dissolve in the saliva and then swallowed without the need for water. FDT's are not only indicated for people who have swallowing difficulties, but also are ideal for active people. FDT's are those when put on tongue disintegrates instantaneously releasing the drug, which dissolves or disperse in saliva ${ }^{1}$.

Salbutamol sulphate is a short-acting B2-adrenergic receptor agonist used for the relief of bronchospasm in conditions such as asthma and COPD. (Chronic Obstructive Pulmonary Disease). Salbutamol is well absorbed from the gastrointestinal tract having 50\% oral bioavailability due to first pass metabolism. Hence an attempt was made for preparing fast dissolving tablet of salbutamol sulphate and compares along with natural and synthetic superdisintegrants. With an aim of reducing the lag time and providing faster onset of action to relieve immediately acute asthmatic attack. This would be advantageous as conventional solid oral dosage forms are often associated with first pass effect, longer lag time and slower onset of action but require careful handling. Aerosol systems are specific but fail to deliver actual dose of drug with only $10 \%$ of administered dose deposited on the bronchi while rest of the drug is deposited in oropharynx and is swallowed. Also, metered dose system are less potable while dry powder inhalers cause clogging of device and require skilful operation. FDT's would be advantageous, as salbutamol sulphate is water soluble and its preparation into FDT's would render it to dissolve rapidly and thereby result in rapid absorption improved oral bioavailability without any lag time ${ }^{2}$.

Various natural substances have been used in the formulations of FDT's. Mucilage of natural origin is ¿ 2011, JDDT. All Rights Reserved preferred over semi-synthetic and synthetic substances because they are comparatively cheaper, abundantly available, non-irritating and nontoxicin nature. In the present investigation, the preparation and evaluation of fast dissolving tablets by using different concentrations of natural superdisintegrant that is Plantago ovata mucilage is studied. The reasons for selection of Plantago ovata mucilage because it's high swelling index. Mucilage of Plantago ovata has various characteristics like binding, disintegrating and sustaining properties. Hence, in present study, mucilage of Plantago ovata was used to develop FDT's of salbutamol sulphate. The concept of formulating FDT's of salbutamol sulphate increases the water uptake with shortest wetting time and there by decrease the disintegration time of the tablets by simple and cost effective direct compression techniques ${ }^{3,4}$.

\section{MATERIALS AND METHODS}

\section{Materials:}

Salbutamol Sulphate was gifted by Glenmark Pharmaceuticals Ltd. Nashik. Plantago ovata seeds were purchased from local market Mumbai. Plantago ovata husk powder was obtained as a gift sample from Gayatri Psyllium husk powder, Unjha Gujarat. Crosscarmellose sodium, Sodium starch glycolate, Microcrystalline cellulose, Mannitol, Colloidal silicon dioxide, Talc, Aspartame and Vanillin used were of analytical grades.

\section{Methods:}

Formulation of FDT's by direct compression method:

Salbutamol sulphate, directly compressible (microcrystalline cellulose), superdisintegrants (POM/POH/croscarmellose sodium/sodium starch glycolate), colloidal silicon dioxide, mannitol, were sifted ISSN: 2250-1177 
through the sieve \#44 and admixed for about 15 minutes to make a uniform blend. Talc, aspartame, vanillin were passed through sieve \#100 and mixed with the above blend for approximately 5-7 minutes. The blend was evaluated for precompression parameters. The resulting uniform blends were directly compressed using $6 \mathrm{~mm}$, round convex faced tooling to make the tablets using 10 station compression machine (Rimek, Mini Press-1, Karnavati Engineering Limited). The tablet press setting was kept constant across all formulations.

\section{STANDARD CALIBRATION CURVE}

The solutions of salbutamol sulphate in the range of 60$100 \mu \mathrm{g} / \mathrm{ml}$ and $20-100 \mu \mathrm{g} / \mathrm{ml}$ in phosphate buffer $\mathrm{pH} 6.8$ and distilled water were prepared respectively. Absorbance was measured for each solution at $\lambda \max$ of $276.4 \mathrm{~nm}$ and $275 \mathrm{~nm}$ respectively, using UV-Vis Spectrophotometer (Jasco- V630, Japan).

\section{AUTHENTICATION OF Plantago ovata SEEDS}

The seeds of Plantago ovata were authenticated from Botany Department NDMVP's junior college, Nashik. The authentication results proves that the seeds are of Plantago ovata belonging to family Plantaginaceae.

\section{METHODOLOGY FOR ISOLATION OF MUCILAGE}

Seeds of Plantago ovata were soaked in distilled water for $48 \mathrm{hrs}$. Soaked seeds were boiled for 120 minutes till mucilage get released into the water completely. As soon as mucilage gets released the mucilage was squeezed out and separated from seeds with the help of nylon muslin cloth. The mucilage collected and precipitated using 95\% ethanol (1:2) and washed twice with the same. Collected mucilage was dried in the tray dryer at $50-55^{\circ} \mathrm{C}$. Dried mucilage was scraped and powdered using mortar and pestle. This crushed and fine powder was then passed through \# 80. The mucilage was then kept in dessicator until its use ${ }^{5}$.

Physicochemical and Phytochemical evaluation of dried powdered mucilage $e^{6,7,8}$

\section{Organoleptic properties}

Organoleptic properties such as physical appearance, colour, odour and taste of dried powdered mucilage were determined.

\section{Solubility profile}

The solubility of dried powdered mucilage was checked by adding a pinch in the solvent such as water.

\section{Charring}

A few milligrams of dried mucilage powder and husk powder were placed in a melting-point apparatus. The temperature was taken and recorded when the material started to char.

\section{pH determination}

The $\mathrm{pH}$ values of solutions prepared in specified strength were determined using calibrated $(\mathrm{pH} 4$ and $\mathrm{pH}$ 7) digital $\mathrm{pH}$ meter.

Preliminary phytochemical screening
A preliminary phytochemical screening of dried powdered mucilage was carried out for the detection of various phytoconstituents.

\section{Ash values (WHO GUIDELINES)}

Total ash, acid-insoluble ash, water-soluble ash, sulphated ash values were evaluated.

\section{Loss on Drying}

Loss on Drying was determined for an appropriate quantity of dried powdered mucilage at $105^{\circ} \mathrm{C}$ for 5 hours.

LOD $(\%)=($ Wt of water in sample/Wt of dry sample) $x 100$

\section{Swelling power}

The swelling power of the dried mucilage powder was performed as outlined in Indian Pharmacopoeia.

\section{Viscosity determination}

Rheological studies of dried mucilage were carried out using varying concentrations $(0.1-0.5 \%$ v $)$ prepared in distilled water. The viscosities were measured using a Brookfield viscometer spindle no. 62 at $100 \mathrm{rpm}$ at $25^{\circ} \mathrm{C}$.

Microbial count

The microbial count of the dried mucilage powder was performed as outlined in Indian Pharmacopoeia for the presence of bacteria as well as for fungi. Total count of bacteria and fungi was calculated using plate count method.

Particle size determination

The particle size of the dried-powder mucilage and husk powder were determined by the microscopic method.

\section{Hydation capacity (water retention capacity)}

$1 \mathrm{gm}$ of dried-powder mucilage was placed in a centrifuge tube and covered with $10 \mathrm{~mL}$ of distilled water. The tube was shaken intermittently over 2 hours and left to stand for 10 minutes at $3000 \mathrm{rpm}$. With the supernatant decanted and the weight of the powder after water uptake and centrifugation, $\mathbf{x}$ was determined.

$$
\text { Hydration capacity }=x / y
$$

Where $\mathbf{x}$ is the weight of moist powder after centrifugation and $\mathbf{y}$ is the weight of dry powder respectively.

Flow properties of dried mucilage powder

The flow properties of dried mucilage powder were characterized.

\section{Drug excipients Compatibility studies}

\section{Fourier Transform Infra-Red Spectroscopy (FTIR)}

The drug and physical mixture of drug and excipients were subjected to IR spectroscopic study using FT-IR spectrophotometer (Bruker Alpha ATR).

\section{Differential Scanning Calorimetry}

Thermogram of drug and physical mixture (drug: POM); (ratio1:5) were employed (DSC60 SHIMADZU) for the determination of glass transition temperature $(\mathrm{Tg})$.

\section{Assignment of formulation code}


Various formulations of Salbutamol sulphate (API) fast dissolving tablets(FDTs) were designed utilizing natural superdisintegrants as mucilage of Plantago ovata (POM), husk of Plantago ovata (POH) and synthetic superdisintegrants as Crosscarmelose $\operatorname{sodium}(\mathrm{CCS})$, Sodium starch glycolate (SSG) each varied at different concentrations $(2,4,6,8$ and $10 \%)$. All of the other ingredients were kept constant. A total of such fifteen formulations prepared were designated with their codes and will be referred with the same in further sections. The assigned formulation codes were as follows: SM1, SM2, SM3, SM4 and SM5 for formulations containing mucilage of Plantago ovata and SH1, SH2, SH3, SH4 and SH5 for formulations containing husk of Plantago ovata as a superdisintegrant with concentrations 2, 4, 6, 8 and $10 \%$ respectively. Similarly, SC1, SC2, SC3, SC4 and SC5 also SS1, SS2, SS3, SS4 and SS5 were the assigned codes for the formulations prepared with crosscarmellose sodium, Sodium starch glycolate as superdisintegrants at the percentage levels provided for PO above.

\section{Evaluation of fast dissolving tablets ${ }^{9,10}$}

\section{Weight variation}

Twenty tablets were selected randomly from each formulation and weighed individually. The individual weights were compared with the average weight for the weight variation.

\section{Hardness and Friability}

Hardness of the tablets was measured using the Monsanto hardness tester. The friability of a sample of twenty tablets was measured using a USP type Roche friabilator. Preweighed tablets were placed in a plastic chambered friabilator attached to a motor revolving at a speed of 25 rpm for $4 \mathrm{~min}$. The friability was calculated.

\section{Drug content uniformity}

For the content uniformity test, twenty tablets were weighed and pulverized to a fine powder. A quantity of powder equivalent to $4 \mathrm{mg}$ of salbutamol sulphate was taken in $100 \mathrm{ml}$ volumetric flask containing distilled water. An aliquot of $2 \mathrm{ml}$ sample was withdrawn and diluted to $10 \mathrm{ml}$ and analysed by UV spectrophotometer at $275 \mathrm{~nm}$ against blank. Then the amount of drug present was calculater using standard calibration curve. The mean percent drug content was calculated as an average of three determinations.

\section{In vitro disintegration time}

Six tablets were placed individually in each tube of disintegration test apparatus and discs were placed. Distilled water was maintained at a temperature of $37^{\circ} \pm 2^{\circ} \mathrm{C}$ and time taken for the entire tablet to disintegrate completely was noted.

\section{Wetting time and water absorption ratio ( $R$ )}

Twice folded tissue paper was placed in a Petri dish having an internal diameter of $6.5 \mathrm{~cm}$ to that added $6 \mathrm{ml}$ of purified water. A tablet was carefully placed on the surface of the tissue paper in the Petri dish. The time required for water to reach the upper surface of the tablet and to completely wet it was noted as the wetting time. Water absorption ratio $(\mathrm{R})$ was then determined according to the following equation:

$$
R=[(W a-W b) / W b] x 100
$$

Where; $\mathrm{Wb}$ and $\mathrm{Wa}$ were tablet weights before and after water absorption, respectively.

\section{In vitro drug release study}

In vitro dissolution of the tablets was determined using USP- Type-II dissolution test apparatus rotating at $50 \mathrm{rpm}$ in $900 \mathrm{ml} \mathrm{pH6.8} \mathrm{phosphate} \mathrm{buffer} \mathrm{solution} \mathrm{as} \mathrm{medium}$ maintained at $37 \pm 0.5^{\circ} \mathrm{C}$. The amount of drug in solution was determined spectrophotometrically at $276.4 \mathrm{~nm}$.

\section{Stability study}

The fast dissolving tablets were packed in aluminium foil and stored under the following environmental conditions for a period as prescribed by ICH guidelines for accelerated studies at $40^{\circ} \mathrm{C}$ and $75 \% \mathrm{RH}$. The tablets were withdrawn at end of 90 days and evaluated for parameters including disintegration time, drug content and dissolution study.

\section{RESULT AND DISCUSSION}

Phytochemical, microbial and physicochemical characterization of Plantago ovata mucilage

\section{Organoleptic properties}

It is creamish fine powder odourless with mucilaginous taste.

\section{Solubility profile}

Mucilage does not dissolve in water, it swells.

Table 1: Charring and pH determination of dried mucilage powder

\begin{tabular}{|c|c|}
\hline \multicolumn{2}{|c|}{ Charring } \\
\hline Dried mucilage powder & Husk powder \\
\hline $183-184^{\circ} \mathrm{C}$ & $182-183^{\circ} \mathrm{C}$ \\
\hline \multicolumn{2}{|c|}{$\mathbf{p H}$ determination } \\
\hline $0.5 \%$ solution & 6.2 \\
\hline
\end{tabular}

Table 2: Preliminary phytochemical screening

\begin{tabular}{|c|c|c|}
\hline \multicolumn{1}{|c|}{ Tests } & Observations & Inferences \\
\hline $\begin{array}{l}\text { Ruthenium test } \\
\text { dried a small quantity of } \\
\text { mount it on a slide with } \\
\text { ruthenium red solution and } \\
\text { observe it under } \\
\text { microscope. }\end{array}$ & $\begin{array}{c}\text { Pink colour } \\
\text { develops }\end{array}$ & $\begin{array}{c}\text { Mucilage } \\
\text { is present }\end{array}$ \\
\hline & & \\
Dried mucilage powder & $\begin{array}{c}\text { Aqueous } \\
\text { potassium } \\
\text { hydroxide }\end{array}$ & Swells \\
\hline
\end{tabular}


The presence of mucilage was confirmed using ruthenium positive. red and aqueous potassium hydroxide. Both tests were

Table 3: Physical properties of dried powder mucilage

\begin{tabular}{|c|c|c|}
\hline Identification tests & Observed results & $\begin{array}{l}\text { Reported standards } \\
\text { (BP 1988, IP 1996) }\end{array}$ \\
\hline Ash value & & Not More Than $4.5 \%$ \\
\hline Total ash $(\%)$ & 3.46 & - \\
\hline Acid-insoluble ash (\%) & 0.33 & Not More Than $0.45 \%$ \\
\hline Water soluble ash (\%) & 2.0 & - \\
\hline Sulphated ash(gm) & 0.149 & - \\
\hline Loss on Drying $(\%)$ & 11.25 & Not More Than $12.0 \%$ determined on $0.5 \mathrm{~g}$ \\
\hline Swelling power(ml) & 42 & Not Less Than $40 \mathrm{ml}$ \\
\hline Viscosity $(\mathrm{cp})$ & 8.42 for $0.3 \%$ solution & - \\
\hline $\begin{array}{l}\text { Microbial count }(\mathrm{cfu} / \mathrm{g}) \\
\text { For bacteria } \\
\text { For fungi }\end{array}$ & $\begin{array}{l}6 \\
3\end{array}$ & - \\
\hline $\begin{array}{l}\text { Particle-size determination }(\mu \mathrm{m}) \\
\text { Dried mucilage powder } \\
\text { Husk powder }\end{array}$ & $\begin{array}{l}150-200 \\
150-200 \\
\end{array}$ & - \\
\hline Hydration capacity & 9.5 & - \\
\hline Bulk density $(\mathrm{g} / \mathrm{ml})$ & 0.49 & - \\
\hline Tapped density (g/ml) & 0.56 & - \\
\hline Carr's index $(\%)$ & 12.745 & - \\
\hline Hausner's ratio & 1.142 & - \\
\hline Angle of repose $\left(^{\circ}\right)$ & 29.05 & - \\
\hline
\end{tabular}

Drug excipients Compatibility studies

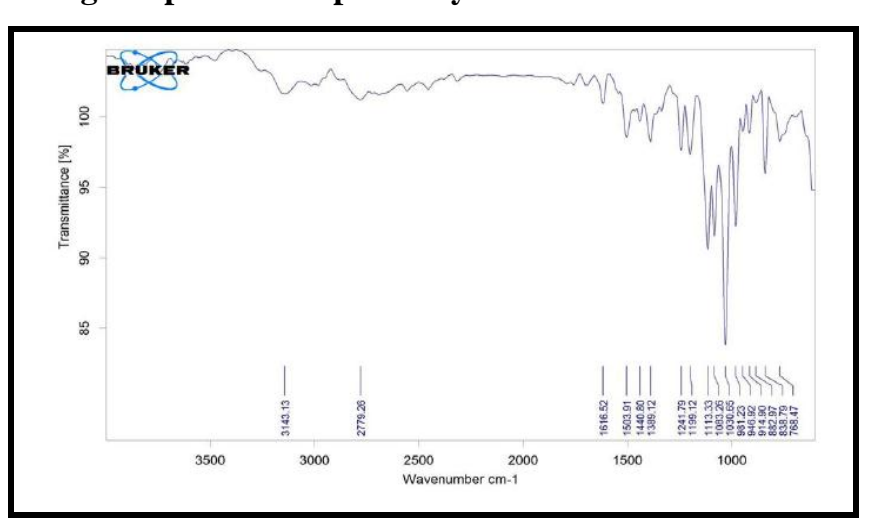

Figure 1: IR Spectrum of Salbutamol sulphate

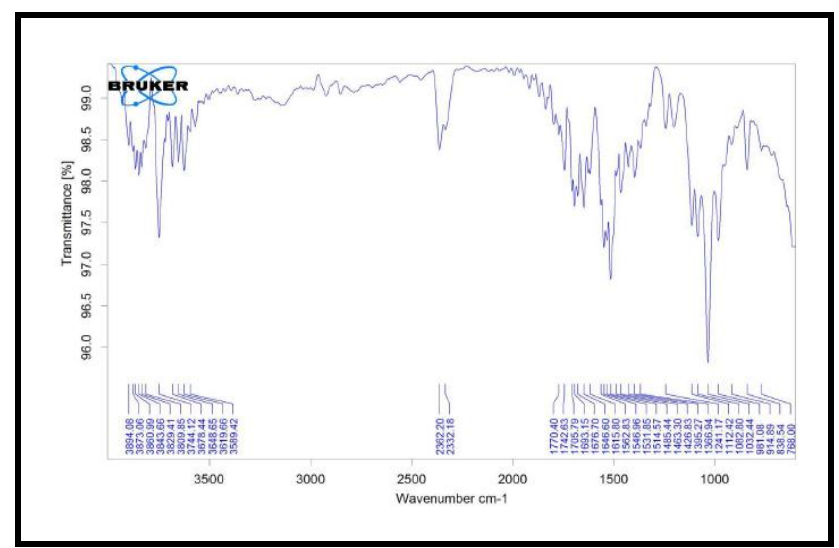

Figure 2: FTIR spectra of Drug + POM

\begin{tabular}{|c|c|c|c|c|}
\hline Sr.No. & Functional groups & Pure drug & POM & Pure drug + POM \\
\hline 1 & C-O(alkyl, aryl) & Yes & Yes & Yes \\
\hline 2 & N=O(aromatic nitroso group) & Yes & Yes & Yes \\
\hline 3 & C-H(bending) & Yes & Yes & Yes \\
\hline 4 & $\begin{array}{c}\text { P=O } \\
\text { (phosphine oxide ) }\end{array}$ & Yes & Yes & Yes \\
\hline 5 & O-H (bending) & Yes & Pes \\
\hline
\end{tabular}

Figure 1 and 2 represents the FTIR spectra of pure drug and drug + POM which were found to contain the same peaks as that found in pure drug, and no any additional peak was observed in physical mixture revealing that no incompatibility exist between them. 


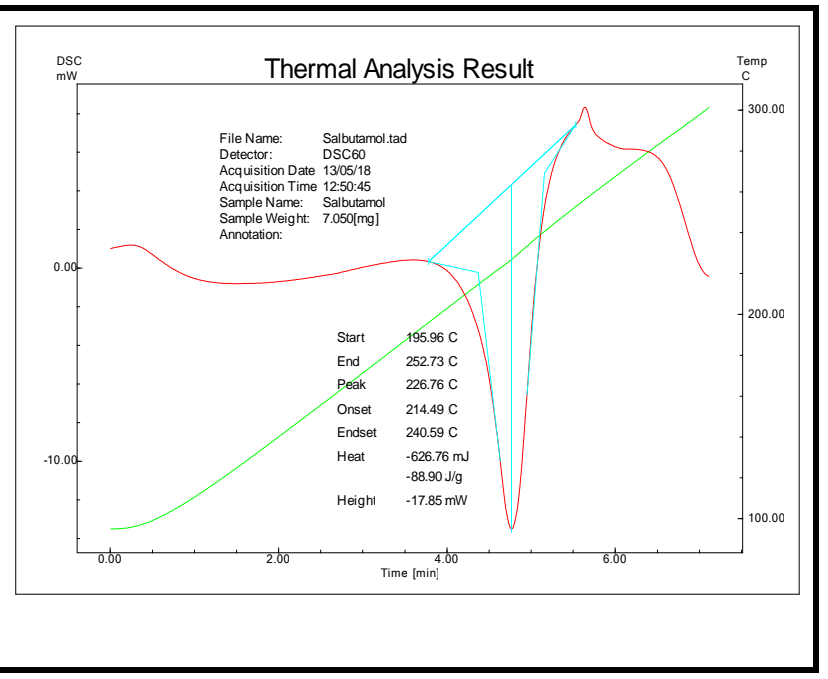

Figure 3: DSC thermogram of Salbutamol sulphate

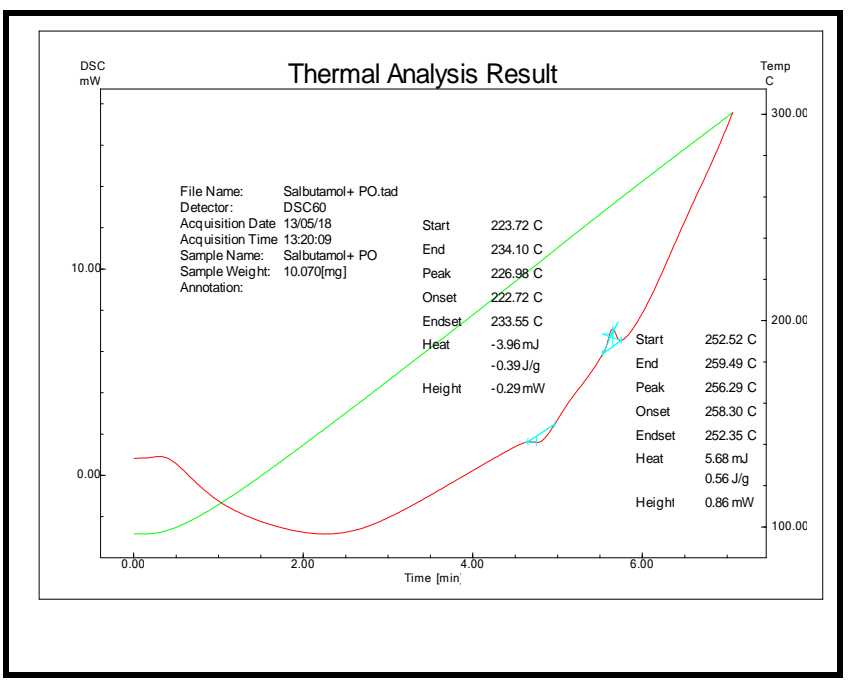

Figure 4: DSC of drug with POM

DSC thermograms of drug and its physical mixture exhibited a sharp endothermic peak at $226.76^{\circ} \mathrm{C}$ and at $226.98^{\circ} \mathrm{C}$ then the intensity was reduced and the peak slightly shifted to $256.29^{\circ} \mathrm{C}$ respectively revealing that no incompatibility exist between them.

Table 4: Composition of formulation batches for fast dissolving tablet of Salbutamol sulphate having weight 100mg

\begin{tabular}{|c|c|c|c|c|c|c|c|c|c|c|c|}
\hline $\begin{array}{c}\text { Ingredients(m } \\
\text { Formulation } \\
\text { Code }\end{array}$ & $\begin{array}{c}\text { Salbutam } \\
\text { ol } \\
\text { Sulphate }\end{array}$ & $\begin{array}{c}\text { PO } \\
\mathbf{M}\end{array}$ & $\begin{array}{c}\text { PO } \\
\mathbf{H}\end{array}$ & $\begin{array}{c}\mathrm{CC} \\
\mathrm{S}\end{array}$ & $\begin{array}{c}\text { SS } \\
\text { G }\end{array}$ & $\begin{array}{c}\text { Tal } \\
\text { c }\end{array}$ & $\begin{array}{c}\text { MC } \\
\mathrm{C}\end{array}$ & $\begin{array}{l}\text { Colloid } \\
\text { al } \mathrm{SiO}_{2}\end{array}$ & $\begin{array}{c}\text { Mannit } \\
\text { ol }\end{array}$ & $\begin{array}{c}\text { Aspartam } \\
\text { e }\end{array}$ & $\begin{array}{c}\text { Vanilli } \\
\mathbf{n}\end{array}$ \\
\hline SM1 & 4 & 2 & - & - & - & 2 & 35 & 0.5 & 50 & 4 & 2 \\
\hline SM2 & 4 & 4 & - & - & - & 2 & 33 & 0.5 & 50 & 4 & 2 \\
\hline SM3 & 4 & 6 & - & - & - & 2 & 31 & 0.5 & 50 & 4 & 2 \\
\hline SM4 & 4 & 8 & - & - & - & 2 & 29 & 0.5 & 50 & 4 & 2 \\
\hline SM5 & 4 & 10 & - & - & - & 2 & 27 & 0.5 & 50 & 4 & 2 \\
\hline SH1 & 4 & - & 2 & - & - & 2 & 35 & 0.5 & 50 & 4 & 2 \\
\hline SH2 & 4 & - & 4 & - & - & 2 & 33 & 0.5 & 50 & 4 & 2 \\
\hline SH3 & 4 & - & 6 & - & - & 2 & 31 & 0.5 & 50 & 4 & 2 \\
\hline SH4 & 4 & - & 8 & - & - & 2 & 29 & 0.5 & 50 & 4 & 2 \\
\hline SH5 & 4 & - & 10 & - & - & 2 & 27 & 0.5 & 50 & 4 & 2 \\
\hline SC1 & 4 & - & - & 2 & - & 2 & 35 & 0.5 & 50 & 4 & 2 \\
\hline SC2 & 4 & - & - & 4 & - & 2 & 33 & 0.5 & 50 & 4 & 2 \\
\hline SC3 & 4 & - & - & 6 & - & 2 & 31 & 0.5 & 50 & 4 & 2 \\
\hline SC4 & 4 & - & - & 8 & - & 2 & 29 & 0.5 & 50 & 4 & 2 \\
\hline SC5 & 4 & - & - & 10 & - & 2 & 27 & 0.5 & 50 & 4 & 2 \\
\hline SS1 & 4 & - & - & - & 2 & 2 & 35 & 0.5 & 50 & 4 & 2 \\
\hline SS2 & 4 & - & - & - & 4 & 2 & 33 & 0.5 & 50 & 4 & 2 \\
\hline SS3 & 4 & - & - & - & 6 & 2 & 31 & 0.5 & 50 & 4 & 2 \\
\hline SS4 & 4 & - & - & - & 8 & 2 & 29 & 0.5 & 50 & 4 & 2 \\
\hline SS5 & 4 & - & - & - & 10 & 2 & 27 & 0.5 & 50 & 4 & 2 \\
\hline
\end{tabular}


Table 5: Evaluation of blend for formulation batches

\begin{tabular}{|c|c|c|c|c|c|c|}
\hline $\begin{array}{l}\text { Formulation } \\
\text { Code }\end{array}$ & $\begin{array}{c}\text { Bulk Density } \\
\text { (g/ml) } \\
\pm \text { S.D }\end{array}$ & $\begin{array}{c}\text { Tapped Density } \\
(\mathrm{g} / \mathrm{ml}) \\
\pm \text { S.D }\end{array}$ & $\begin{array}{c}\text { Carr's } \\
\text { Index (\%) } \\
\pm \text { S.D }\end{array}$ & $\begin{array}{c}\text { Hausner's } \\
\text { Ratio } \\
\pm \text { S.D }\end{array}$ & $\begin{array}{c}\text { Angle of repose } \\
\left({ }^{\circ}\right) \\
\pm \text { S.D }\end{array}$ & $\begin{array}{c}\text { Flow } \\
\text { ability }\end{array}$ \\
\hline SM1 & $0.680 \pm 0.007$ & $0.782 \pm 0.007$ & $12.995 \pm 1.235$ & $1.149 \pm 0.014$ & $24.593 \pm 1.120$ & Excellent \\
\hline SM2 & $0.590 \pm 0.010$ & $0.672 \pm 0.006$ & $12.107 \pm 2.119$ & $1.138 \pm 0.027$ & $23.311 \pm 1.675$ & Excellent \\
\hline SM3 & $0.613 \pm 0.016$ & $0.702 \pm 0.011$ & $12.738 \pm 1.958$ & $1.146 \pm 0.025$ & $23.408 \pm 1.331$ & Excellent \\
\hline SM4 & $0.669 \pm 0.024$ & $0.680 \pm 0.018$ & $11.599 \pm 1.213$ & $1.137 \pm 0.024$ & $24.789 \pm 0.911$ & Excellent \\
\hline SM5 & $0.598 \pm 0.014$ & $0.754 \pm 0.010$ & $11.088 \pm 1.837$ & $1.129 \pm 0.038$ & $21.738 \pm 0.894$ & Excellent \\
\hline SH1 & $0.668 \pm 0.031$ & $0.640 \pm 0.023$ & $10.809 \pm 1.259$ & $1.130 \pm 0.031$ & $24.200 \pm 1.379$ & Excellent \\
\hline $\begin{array}{l}\mathrm{SH} 2 \\
\end{array}$ & $0.567 \pm 0.034$ & $0.629 \pm 0.010$ & $12.895 \pm 1.215$ & $1.125 \pm 0.023$ & $23.436 \pm 0.895$ & Excellent \\
\hline SH3 & $0.618 \pm 0.029$ & $0.682 \pm 0.050$ & $12.117 \pm 2.117$ & $1.136 \pm 0.030$ & $24.962 \pm 1.611$ & Excellent \\
\hline SH4 & $0.553 \pm 0.019$ & $0.783 \pm 0.005$ & $12.748 \pm 1.858$ & $1.121 \pm 0.015$ & $24.485 \pm 1.380$ & Excellent \\
\hline SH5 & $0.608 \pm 0.041$ & $0.674 \pm 0.008$ & $11.597 \pm 1.273$ & $1.142 \pm 0.015$ & $24.475 \pm 1.281$ & Excellent \\
\hline SS1 & $0.681 \pm 0.008$ & $0.712 \pm 0.011$ & $11.098 \pm 1.877$ & $1.134 \pm 0.022$ & $24.216 \pm 1.247$ & Excellent \\
\hline SS2 & $0.588 \pm 0.011$ & $0.634 \pm 0.028$ & $10.819 \pm 1.279$ & $1.123 \pm 0.034$ & $23.418 \pm 1.231$ & Excellent \\
\hline SS3 & $0.611 \pm 0.015$ & $0.753 \pm 0.011$ & $12.985 \pm 1.245$ & $1.128 \pm 0.034$ & $24.769 \pm 0.812$ & Excellent \\
\hline SS4 & $0.665 \pm 0.028$ & $0.643 \pm 0.022$ & $12.117 \pm 2.119$ & $1.127 \pm 0.022$ & $21.758 \pm 0.874$ & Excellent \\
\hline SS5 & $0.596 \pm 0.012$ & $0.627 \pm 0.011$ & $12.728 \pm 1.948$ & $1.144 \pm 0.035$ & $24.201 \pm 1.349$ & Excellent \\
\hline $\mathrm{SC} 1$ & $0.638 \pm 0.034$ & $0.689 \pm 0.053$ & $11.589 \pm 1.113$ & $1.177 \pm 0.027$ & $23.437 \pm 0.875$ & Excellent \\
\hline $\mathrm{SC} 2$ & $0.609 \pm 0.011$ & $0.752 \pm 0.011$ & $11.088 \pm 1.437$ & $1.124 \pm 0.035$ & $24.912 \pm 1.651$ & Excellent \\
\hline $\mathrm{SC} 3$ & $0.661 \pm 0.023$ & $0.641 \pm 0.024$ & $10.809 \pm 1.259$ & $1.120 \pm 0.021$ & $24.475 \pm 1.280$ & Excellent \\
\hline $\mathrm{SC} 4$ & $0.586 \pm 0.011$ & $0.621 \pm 0.011$ & $11.188 \pm 1.837$ & $1.145 \pm 0.024$ & $24.455 \pm 1.381$ & Excellent \\
\hline SC5 & $0.633 \pm 0.032$ & $0.642 \pm 0.051$ & $10.819 \pm 1.259$ & $1.136 \pm 0.021$ & $24.226 \pm 1.217$ & Excellent \\
\hline
\end{tabular}

$( \pm$ S.D represents mean standard deviation)

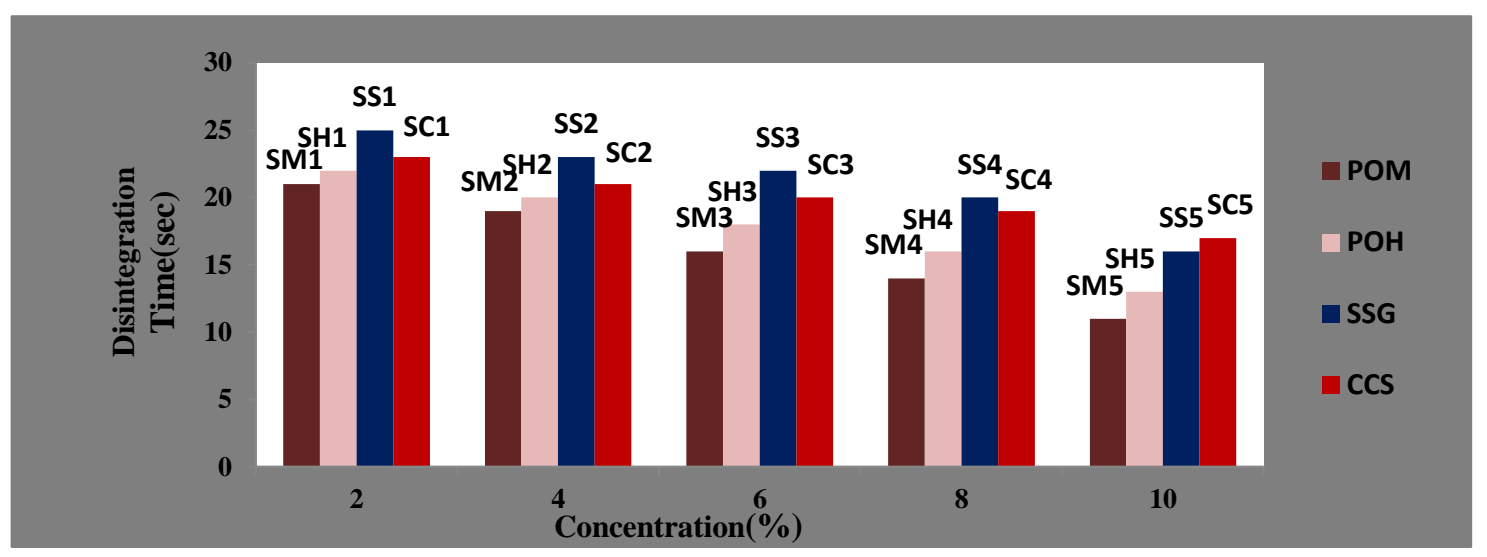

Figure 5: Comparison of natural and synthetic superdisintegrants at different concentrations Vs In vitro disintegration time of tablet 
Pre-compression parameters were within prescribed limits and indicated good free flowing property. Batches which show best results are used for further study.

Here, Friability is less than $1 \%$, Drug content was found to be in the range of 98 to $101 \%$ which is within acceptable limits. Hardness of the tablets was found to be in the range of $2.5-3.0 \mathrm{~kg} / \mathrm{cm}^{2}$. From the result it was found that formulations SM5, SH5, SS5, SC5 shown best results.

Disintegration time of salbutamol sulphate FDT's using mucilage of Plantago ovata, Plantago ovata husk powder, crosscarmellose sodium and sodium starch glycolate as superdisintegrants are shown in Figure 5.

Table 6: Evaluation of compressed tablets for formulation batches

\begin{tabular}{|c|c|c|c|c|c|c|c|c|}
\hline نْ & $\begin{array}{c}\text { Weight } \\
\text { Variation } \\
(\% \\
\text { deviation }) \\
\pm \text { S.D }\end{array}$ & $\begin{array}{c}\text { Hardness } \\
\left(\mathbf{k g} / \mathrm{cm}^{2}\right) \\
\pm \text { S.D }\end{array}$ & $\begin{array}{l}\text { Thickness } \\
\text { (mm) } \\
\pm \text { S.D }\end{array}$ & $\begin{array}{c}\text { Friability } \\
\quad(\%) \\
\pm \text { S.D }\end{array}$ & $\begin{array}{c}\text { In-vitro } \\
\text { DT } \\
\text { (sec) } \\
\pm \text { S.D }\end{array}$ & $\begin{array}{c}\text { Drug } \\
\text { Content } \\
(\%) \\
\pm \text { S.D }\end{array}$ & $\begin{array}{c}\text { Water } \\
\text { Absorption } \\
\text { Ratio (\%) } \\
\pm \text { S.D }\end{array}$ & $\begin{array}{c}\text { Wetting } \\
\text { Time } \\
\text { (sec) } \\
\pm \text { S.D }\end{array}$ \\
\hline SM1 & $\begin{array}{l}98.7 \pm \\
7.40\end{array}$ & $\begin{array}{l}2.53 \pm \\
0.057\end{array}$ & $\begin{array}{l}3.6 \pm \\
0.063\end{array}$ & $\begin{array}{l}0.36 \pm \\
0.007\end{array}$ & $\begin{array}{l}21 \pm \\
1.213\end{array}$ & $\begin{array}{l}98.942 \pm \\
0.12\end{array}$ & $\begin{array}{l}55.46 \pm \\
0.623\end{array}$ & $\begin{array}{l}49 \pm \\
0.164\end{array}$ \\
\hline SM2 & $\begin{array}{l}101 \pm \\
7.6\end{array}$ & $\begin{array}{l}2.56 \pm \\
0.052\end{array}$ & $\begin{array}{l}3.4 \pm \\
0.061\end{array}$ & $\begin{array}{l}0.38 \pm \\
0.006\end{array}$ & $\begin{array}{l}19 \pm \\
1.105 \\
\end{array}$ & $\begin{array}{l}99.891 \pm \\
0.17\end{array}$ & $\begin{array}{l}53.30 \pm \\
0.635 \\
\end{array}$ & $\begin{array}{l}47 \pm \\
0.197\end{array}$ \\
\hline SM3 & $\begin{array}{l}98.9 \pm \\
7.41\end{array}$ & $\begin{array}{l}2.52 \pm \\
0.053\end{array}$ & $\begin{array}{l}3.2 \pm \\
0.065\end{array}$ & $\begin{array}{l}0.34 \pm \\
0.009\end{array}$ & $\begin{array}{l}16 \pm \\
1.132\end{array}$ & $\begin{array}{l}99.242 \pm \\
0.45\end{array}$ & $\begin{array}{l}55.58 \pm \\
0.659\end{array}$ & $\begin{array}{l}45 \pm \\
0.164\end{array}$ \\
\hline SM4 & $\begin{array}{l}98 \pm \\
7.2\end{array}$ & $\begin{array}{l}2.53 \pm \\
0.057\end{array}$ & $\begin{array}{c}3.76 \pm \\
0.067\end{array}$ & $\begin{array}{l}0.256 \pm \\
0.002\end{array}$ & $\begin{array}{l}14.67 \pm \\
1.154\end{array}$ & $\begin{array}{l}99.64 \pm \\
0.82\end{array}$ & $\begin{array}{l}55.25 \pm \\
0.138\end{array}$ & $\begin{array}{l}43 \pm \\
2.645\end{array}$ \\
\hline SM5 & $\begin{array}{l}102 \pm \\
7.69\end{array}$ & $\begin{array}{l}2.55 \pm \\
0.061\end{array}$ & $\begin{array}{l}3.7 \pm \\
0.066\end{array}$ & $\begin{array}{l}0.39 \pm \\
0.015\end{array}$ & $\begin{array}{l}12 \pm \\
1.152\end{array}$ & $\begin{array}{l}98.212 \pm \\
0.23\end{array}$ & $\begin{array}{l}55.56 \pm \\
0.432\end{array}$ & $\begin{array}{l}42 \pm \\
0.136\end{array}$ \\
\hline SH1 & $\begin{array}{l}99.05 \pm \\
7.42\end{array}$ & $\begin{array}{l}2.56 \pm \\
0.059\end{array}$ & $\begin{array}{l}3.5 \pm \\
0.059\end{array}$ & $\begin{array}{l}0.35 \pm \\
0.016\end{array}$ & $\begin{array}{l}22 \pm \\
1.165\end{array}$ & $\begin{array}{l}99.215 \pm \\
0.26\end{array}$ & $\begin{array}{l}54.63 \pm \\
0.645\end{array}$ & $\begin{array}{l}52 \pm \\
0.187 \\
\end{array}$ \\
\hline $\mathrm{SH} 2$ & $\begin{array}{l}98 \pm \\
7.3 \\
\end{array}$ & $\begin{array}{l}2.55 \pm \\
0.051\end{array}$ & $\begin{array}{l}3.9 \pm \\
0.058\end{array}$ & $\begin{array}{l}0.33 \pm \\
0.005\end{array}$ & $\begin{array}{l}20 \pm \\
1.134 \\
\end{array}$ & $\begin{array}{l}98.220 \pm \\
0.29\end{array}$ & $\begin{array}{l}53.54 \pm \\
0.635\end{array}$ & $\begin{array}{l}50 \pm \\
0.465\end{array}$ \\
\hline SH3 & $\begin{array}{l}101 \pm \\
7.5\end{array}$ & $\begin{array}{l}2.59 \pm \\
0.056\end{array}$ & $\begin{array}{l}3.3 \pm \\
0.056\end{array}$ & $\begin{array}{l}0.38 \pm \\
0.042\end{array}$ & $\begin{array}{l}18 \pm \\
1.132\end{array}$ & $\begin{array}{l}98.221 \pm \\
0.26\end{array}$ & $\begin{array}{l}53.62 \pm \\
0.125\end{array}$ & $\begin{array}{l}48 \pm \\
0.152\end{array}$ \\
\hline SH4 & $\begin{array}{l}100 \pm \\
7.42\end{array}$ & $\begin{array}{l}2.56 \pm \\
0.057\end{array}$ & $\begin{array}{l}3.86 \pm \\
0.057\end{array}$ & $\begin{array}{l}0.461 \pm \\
0.002\end{array}$ & $\begin{array}{l}16.33 \pm \\
1.154\end{array}$ & $\begin{array}{c}99.27 \pm \\
0.91\end{array}$ & $\begin{array}{l}54.23 \pm \\
0.703\end{array}$ & $\begin{array}{l}46 \pm \\
0.215\end{array}$ \\
\hline SH5 & $\begin{array}{l}98.2 \pm \\
7.36\end{array}$ & $\begin{array}{l}2.63 \pm \\
0.054\end{array}$ & $\begin{array}{l}3.4 \pm \\
0.063\end{array}$ & $\begin{array}{l}0.27 \pm \\
0.003\end{array}$ & $\begin{array}{l}14 \pm \\
1.145\end{array}$ & $\begin{array}{l}98.721 \pm \\
0.26\end{array}$ & $\begin{array}{l}53.45 \pm \\
0.345\end{array}$ & $\begin{array}{l}44 \pm \\
0.175\end{array}$ \\
\hline SS1 & $\begin{array}{l}98 \pm \\
7.41\end{array}$ & $\begin{array}{l}2.69 \pm \\
0.058\end{array}$ & $\begin{array}{l}3.9 \pm \\
0.065\end{array}$ & $\begin{array}{l}0.29 \pm \\
0.019\end{array}$ & $\begin{array}{l}25 \pm \\
1.123\end{array}$ & $\begin{array}{l}97.210 \pm \\
0.53\end{array}$ & $\begin{array}{l}54.54 \pm \\
0.345\end{array}$ & $\begin{array}{l}73 \pm \\
0.365\end{array}$ \\
\hline SS2 & $\begin{array}{l}100 \pm \\
7.43\end{array}$ & $\begin{array}{l}2.54 \pm \\
0.062\end{array}$ & $\begin{array}{l}2.9 \pm \\
0.064\end{array}$ & $\begin{array}{l}0.31 \pm \\
0.015\end{array}$ & $\begin{array}{l}23 \pm \\
1.102\end{array}$ & $\begin{array}{l}98.212 \pm \\
0.65\end{array}$ & $\begin{array}{l}55.00 \pm \\
0.345\end{array}$ & $\begin{array}{l}71 \pm \\
0.145\end{array}$ \\
\hline SS3 & $\begin{array}{l}99 \pm \\
7.42\end{array}$ & $\begin{array}{l}2.47 \pm \\
0.064\end{array}$ & $\begin{array}{l}3.1 \pm \\
0.066\end{array}$ & $\begin{array}{l}0.28 \pm \\
0.005\end{array}$ & $\begin{array}{l}20 \pm \\
1.131 \\
\end{array}$ & $\begin{array}{l}98.450 \pm \\
0.26\end{array}$ & $\begin{array}{l}54.64 \pm \\
0.659 \\
\end{array}$ & $\begin{array}{l}69 \pm \\
0.136 \\
\end{array}$ \\
\hline SS4 & $\begin{array}{l}99 \pm \\
7.43\end{array}$ & $\begin{array}{l}2.56 \pm \\
0.057\end{array}$ & $\begin{array}{l}3.76 \pm \\
0.057\end{array}$ & $\begin{array}{l}0.153 \pm \\
0.001\end{array}$ & $\begin{array}{l}18.67 \pm \\
1.52\end{array}$ & $\begin{array}{l}99.15 \pm \\
0.81\end{array}$ & $\begin{array}{l}55.213 \pm \\
0.114\end{array}$ & $\begin{array}{l}64 \pm \\
0.154\end{array}$ \\
\hline SS5 & $\begin{array}{l}98.7 \pm \\
7.40\end{array}$ & $\begin{array}{l}2.53 \pm \\
0.063\end{array}$ & $\begin{array}{l}3.9 \pm \\
0.059\end{array}$ & $\begin{array}{l}0.26 \pm \\
0.004\end{array}$ & $\begin{array}{l}16 \pm \\
1.326 \\
\end{array}$ & $\begin{array}{l}98.254 \pm \\
0.28\end{array}$ & $\begin{array}{l}55.64 \pm \\
0.263\end{array}$ & $\begin{array}{l}59 \pm \\
0.132\end{array}$ \\
\hline SC1 & $\begin{array}{l}98 \pm \\
7.41\end{array}$ & $\begin{array}{l}2.58 \pm \\
0.052\end{array}$ & $\begin{array}{l}3.4 \pm \\
0.061\end{array}$ & $\begin{array}{l}0.37 \pm \\
0.006\end{array}$ & $\begin{array}{l}23 \pm \\
1.147\end{array}$ & $\begin{array}{l}98.654 \pm \\
0.27\end{array}$ & $\begin{array}{l}54.52 \pm \\
0.645\end{array}$ & $\begin{array}{l}54 \pm \\
0.135\end{array}$ \\
\hline SC2 & $\begin{array}{l}99 \pm \\
7.42\end{array}$ & $\begin{array}{l}2.65 \pm \\
0.061\end{array}$ & $\begin{array}{l}3.6 \pm \\
0.063\end{array}$ & $\begin{array}{l}0.45 \pm \\
0.009\end{array}$ & $\begin{array}{l}21 \pm \\
1.132\end{array}$ & $\begin{array}{l}98.214 \pm \\
0.42\end{array}$ & $\begin{array}{l}55.62 \pm \\
0.152\end{array}$ & $\begin{array}{l}53 \pm \\
0.132\end{array}$ \\
\hline SC3 & $\begin{array}{l}101 \pm \\
7.57\end{array}$ & $\begin{array}{l}2.56 \pm \\
0.049\end{array}$ & $\begin{array}{l}3.5 \pm \\
0.065\end{array}$ & $\begin{array}{l}0.48 \pm \\
0.016\end{array}$ & $\begin{array}{l}19 \pm \\
1.156\end{array}$ & $\begin{array}{l}99.254 \pm \\
0.46\end{array}$ & $\begin{array}{l}55.66 \pm \\
0.356\end{array}$ & $\begin{array}{l}51 \pm \\
0.215\end{array}$ \\
\hline SC4 & $\begin{array}{l}99 \pm \\
7.42\end{array}$ & $\begin{array}{l}2.66 \pm \\
0.057\end{array}$ & $\begin{array}{l}3.83 \pm \\
0.057\end{array}$ & $\begin{array}{l}0.35 \pm \\
0.0152\end{array}$ & $\begin{array}{l}16.33 \pm \\
1.154\end{array}$ & $\begin{array}{c}99.12 \pm \\
1.045\end{array}$ & $\begin{array}{l}56.71 \pm \\
0.762\end{array}$ & $\begin{array}{l}49 \pm \\
0.154\end{array}$ \\
\hline SC5 & $\begin{array}{l}101 \pm \\
7.57\end{array}$ & $\begin{array}{l}2.54 \pm \\
0.053\end{array}$ & $\begin{array}{l}2.9 \pm \\
0.059\end{array}$ & $\begin{array}{l}0.44 \pm \\
0.006\end{array}$ & $\begin{array}{l}14 \pm \\
1.152\end{array}$ & $\begin{array}{l}98.247 \pm \\
0.49\end{array}$ & $\begin{array}{l}54.66 \pm \\
0.236\end{array}$ & $\begin{array}{l}47 \pm \\
0.214\end{array}$ \\
\hline
\end{tabular}

( \pm S.D represents mean standard deviation)

\section{Comparative Dissolution Profiles of all the formulations}

The in-vitro dissolution profiles of prepared tablets are shown in Table 7. 
Table 7: Comparative Dissolution Profiles of all the formulations

\begin{tabular}{|c|c|c|c|c|c|c|}
\hline$\underbrace{\text { Time }(\min )}_{\text {Formulations }}$ & $\begin{array}{c}\mathbf{1} \\
\pm \text { S.D }\end{array}$ & $\begin{array}{r}\mathbf{2} \\
\pm \text { S.D } \\
\end{array}$ & $\begin{array}{c}\mathbf{4} \\
+ \text { S.D }\end{array}$ & $\begin{array}{c}6 \\
\pm \text { S.D }\end{array}$ & $\begin{array}{c}\mathbf{8} \\
\pm \text { S.D }\end{array}$ & $\begin{array}{c}\mathbf{1 0} \\
\pm \text { S.D }\end{array}$ \\
\hline SM1 & $\begin{array}{c}74.23 \pm \\
0.11\end{array}$ & $\begin{array}{c}78.86 \pm \\
0.16\end{array}$ & $\begin{array}{c}82.24 \pm \\
0.21\end{array}$ & $\begin{array}{c}86.34 \pm \\
0.24\end{array}$ & $\begin{array}{c}91.32 \pm \\
0.26\end{array}$ & $\begin{array}{c}94.97 \pm \\
0.31\end{array}$ \\
\hline SM2 & $\begin{array}{c}75.36 \pm \\
0.13 \\
\end{array}$ & $\begin{array}{c}80.72 \pm \\
0.11 \\
\end{array}$ & $\begin{array}{c}83.31 \pm \\
0.16 \\
\end{array}$ & $\begin{array}{c}87.46 \pm \\
0.15 \\
\end{array}$ & $\begin{array}{c}92.23 \pm \\
0.31 \\
\end{array}$ & $\begin{array}{c}95.48 \pm \\
0.33\end{array}$ \\
\hline SM3 & $\begin{array}{c}76.98 \pm \\
0.14\end{array}$ & $\begin{array}{c}80.23 \pm \\
0.14\end{array}$ & $\begin{array}{c}84.63 \pm \\
0.13\end{array}$ & $\begin{array}{c}88.43 \pm \\
0.17\end{array}$ & $\begin{array}{c}93.62 \pm \\
0.12\end{array}$ & $\begin{array}{c}96.32 \pm \\
0.21\end{array}$ \\
\hline SM4 & $\begin{array}{c}77.87 \pm \\
0.22\end{array}$ & $\begin{array}{c}79.93 \pm \\
0.16\end{array}$ & $\begin{array}{c}85.14 \pm \\
0.31\end{array}$ & $\begin{array}{c}89.42 \pm \\
0.24\end{array}$ & $\begin{array}{c}94.31 \pm \\
0.31\end{array}$ & $\begin{array}{c}97.62 \pm \\
0.31\end{array}$ \\
\hline SM5 & $\begin{array}{c}78.27 \pm \\
0.12\end{array}$ & $\begin{array}{c}81.36 \pm \\
0.21\end{array}$ & $\begin{array}{c}86.21 \pm \\
0.16\end{array}$ & $\begin{array}{c}91.47 \pm \\
0.11\end{array}$ & $\begin{array}{c}95.21 \pm \\
0.31\end{array}$ & $\begin{array}{c}99.43 \pm \\
0.32\end{array}$ \\
\hline SH1 & $\begin{array}{c}73.23 \pm \\
0.16\end{array}$ & $\begin{array}{c}77.76 \pm \\
0.12\end{array}$ & $\begin{array}{c}81.21 \pm \\
0.15\end{array}$ & $\begin{array}{c}87.83 \pm \\
0.16\end{array}$ & $\begin{array}{c}90.32 \pm \\
0.38\end{array}$ & $\begin{array}{c}92.47 \pm \\
0.16\end{array}$ \\
\hline $\mathrm{SH} 2$ & $\begin{array}{c}75.67 \pm \\
0.17\end{array}$ & $\begin{array}{c}78.97 \pm \\
0.16\end{array}$ & $\begin{array}{c}82.13 \pm \\
0.25\end{array}$ & $\begin{array}{c}88.42 \pm \\
0.21\end{array}$ & $\begin{array}{c}92.46 \pm \\
0.32\end{array}$ & $\begin{array}{c}94.84 \pm \\
0.18\end{array}$ \\
\hline SH3 & $\begin{array}{c}76.43 \pm \\
0.11\end{array}$ & $\begin{array}{c}79.72 \pm \\
0.17\end{array}$ & $\begin{array}{c}82.42 \pm \\
0.21\end{array}$ & $\begin{array}{c}88.81 \pm \\
0.21\end{array}$ & $\begin{array}{c}93.12 \pm \\
0.34\end{array}$ & $\begin{array}{c}95.44 \pm \\
0.31\end{array}$ \\
\hline SH4 & $\begin{array}{c}76.61 \pm \\
0.21 \\
\end{array}$ & $\begin{array}{c}80.76 \pm \\
0.23 \\
\end{array}$ & $\begin{array}{c}82.83 \pm \\
0.24 \\
\end{array}$ & $\begin{array}{c}88.94 \pm \\
0.31 \\
\end{array}$ & $\begin{array}{c}93.42 \pm \\
0.24 \\
\end{array}$ & $\begin{array}{c}96.48 \pm \\
0.34\end{array}$ \\
\hline SH5 & $\begin{array}{c}77.21 \pm \\
0.11\end{array}$ & $\begin{array}{c}83.88 \pm \\
0.16\end{array}$ & $\begin{array}{c}86.11 \pm \\
0.35\end{array}$ & $\begin{array}{c}89.42 \pm \\
0.31\end{array}$ & $\begin{array}{c}94.47 \pm \\
0.32\end{array}$ & $\begin{array}{c}98.83 \pm \\
0.24\end{array}$ \\
\hline SS1 & $\begin{array}{c}74.46 \pm \\
0.13\end{array}$ & $\begin{array}{c}76.23 \pm \\
0.13\end{array}$ & $\begin{array}{c}81.42 \pm \\
0.25\end{array}$ & $\begin{array}{c}85.66 \pm \\
0.35\end{array}$ & $\begin{array}{c}91.23 \pm \\
0.32\end{array}$ & $\begin{array}{c}94.69 \pm \\
0.24\end{array}$ \\
\hline SS2 & $\begin{array}{c}75.42 \pm \\
0.11\end{array}$ & $\begin{array}{c}77.21 \pm \\
0.21\end{array}$ & $\begin{array}{c}82.42 \pm \\
0.21\end{array}$ & $\begin{array}{c}87.98 \pm \\
0.31\end{array}$ & $\begin{array}{c}93.42 \pm \\
0.21\end{array}$ & $\begin{array}{c}96.69 \pm \\
0.21\end{array}$ \\
\hline SS3 & $\begin{array}{c}76.62 \pm \\
0.16\end{array}$ & $\begin{array}{c}79.21 \pm \\
0.16\end{array}$ & $\begin{array}{c}82.89 \pm \\
0.14\end{array}$ & $\begin{array}{c}88.21 \pm \\
0.16\end{array}$ & $\begin{array}{c}94.49 \pm \\
0.19\end{array}$ & $\begin{array}{c}97.21 \pm \\
0.22\end{array}$ \\
\hline SS4 & $\begin{array}{c}77.29 \pm \\
0.26\end{array}$ & $\begin{array}{c}80.84 \pm \\
0.24\end{array}$ & $\begin{array}{c}83.21 \pm \\
0.24\end{array}$ & $\begin{array}{c}89.62 \pm \\
0.24\end{array}$ & $\begin{array}{c}95.12 \pm \\
0.22\end{array}$ & $\begin{array}{c}98.89 \pm \\
0.23\end{array}$ \\
\hline SS5 & $\begin{array}{c}78.92 \pm \\
0.11\end{array}$ & $\begin{array}{c}82.05 \pm \\
0.15\end{array}$ & $\begin{array}{c}86.87 \pm \\
0.16\end{array}$ & $\begin{array}{c}91.21 \pm \\
0.21\end{array}$ & $\begin{array}{c}97.98 \pm \\
0.23\end{array}$ & $\begin{array}{c}98.92 \pm \\
0.31\end{array}$ \\
\hline $\mathrm{SC} 1$ & $\begin{array}{c}73.73 \pm \\
0.16\end{array}$ & $\begin{array}{c}76.21 \pm \\
0.22\end{array}$ & $\begin{array}{c}81.58 \pm \\
0.31\end{array}$ & $\begin{array}{c}84.76 \pm \\
0.31\end{array}$ & $\begin{array}{c}89.82 \pm \\
0.38\end{array}$ & $\begin{array}{c}94.97 \pm \\
0.35\end{array}$ \\
\hline $\mathrm{SC} 2$ & $\begin{array}{c}75.17 \pm \\
0.13\end{array}$ & $\begin{array}{c}79.22 \pm \\
0.35\end{array}$ & $\begin{array}{c}84.56 \pm \\
0.32\end{array}$ & $\begin{array}{c}87.89 \pm \\
0.42\end{array}$ & $\begin{array}{c}91.92 \pm \\
0.41\end{array}$ & $\begin{array}{c}96.01 \pm \\
0.42\end{array}$ \\
\hline $\mathrm{SC} 3$ & $\begin{array}{c}76.38 \pm \\
0.24\end{array}$ & $\begin{array}{c}80.92 \pm \\
0.16\end{array}$ & $\begin{array}{c}85.43 \pm \\
0.35\end{array}$ & $\begin{array}{c}88.56 \pm \\
0.32\end{array}$ & $\begin{array}{c}92.66 \pm \\
0.36\end{array}$ & $\begin{array}{c}97.72 \pm \\
0.25\end{array}$ \\
\hline SC4 & $\begin{array}{c}77.69 \pm \\
0.11\end{array}$ & $\begin{array}{c}81.74 \pm \\
0.39\end{array}$ & $\begin{array}{c}86.92 \pm \\
0.31\end{array}$ & $\begin{array}{c}89.15 \pm \\
0.36\end{array}$ & $\begin{array}{c}93.33 \pm \\
0.34\end{array}$ & $\begin{array}{c}98.45 \pm \\
0.37\end{array}$ \\
\hline SC5 & $\begin{array}{c}77.93 \pm \\
0.12\end{array}$ & $\begin{array}{c}81.96 \pm \\
0.11\end{array}$ & $\begin{array}{c}87.23 \pm \\
0.21\end{array}$ & $\begin{array}{c}91.76 \pm \\
0.21\end{array}$ & $\begin{array}{c}94.21 \pm \\
0.15\end{array}$ & $\begin{array}{c}98.97 \pm \\
0.25\end{array}$ \\
\hline
\end{tabular}

( \pm S.D represents mean standard deviation)

The graphical representation of in-vitro dissolution profiles of prepare tablets are shown in Figure 6 and 7.

1) Comparative dissolution profiles of comparable formulations SM5, SH5, SC5 and SS5.

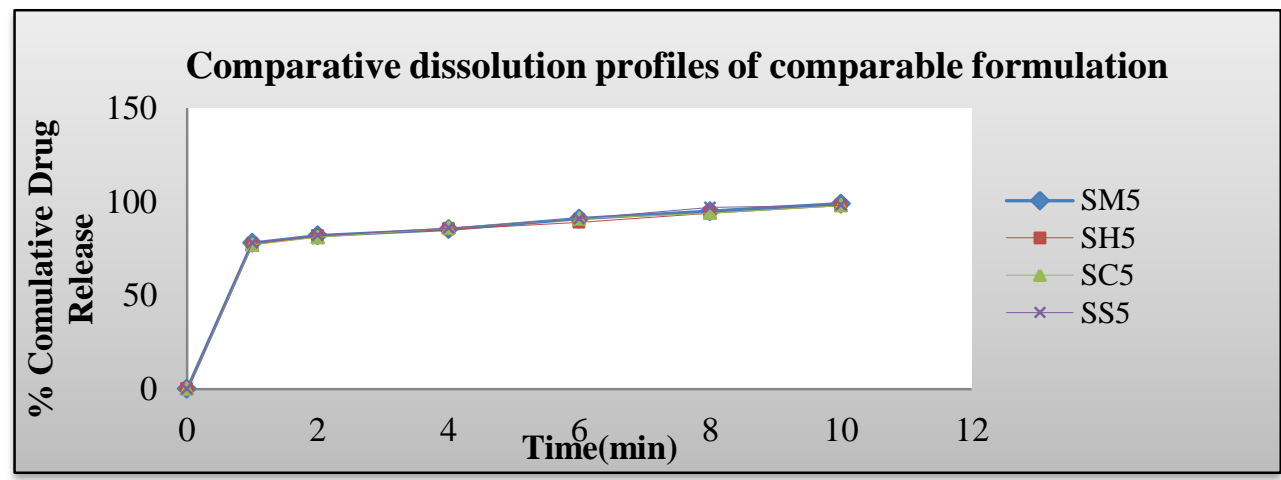

Figure 6: Comparative dissolution profiles of comparable formulations SM5, SH5, SC5 and SS5 
2) Comparative dissolution profiles of all SM1-SM5, SH1-SH5, SS1-SS5, SC1-SC5 formulations

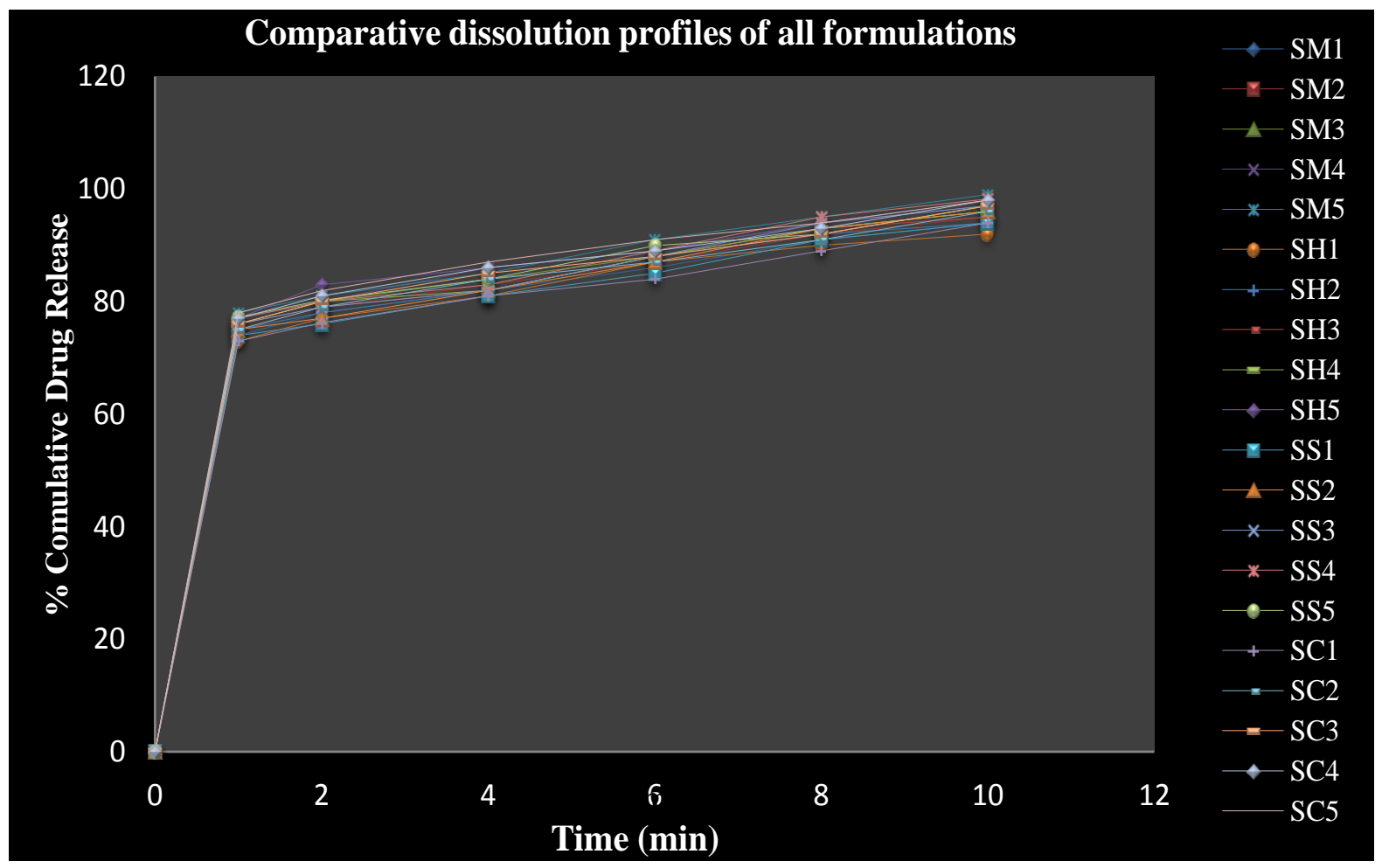

Figure 7: Comparative dissolution profiles of all SM1-SM5, SH1-SH5, SS1-SS5, SC1-SC5 formulations

Among all the designed formulations SM5, SH5, SC5, SS5 was found to be promising and displayed better results. The formulations SM5, SH5 10\% w/w of mucilage of Plantago ovata and husk powder has shown comparable results in all respects than SC5 and SS5 formulations. Invitro dissolution studies on these formulations revealed that more than $90 \%$ drug released within $10 \mathrm{~min}$

\section{Stability studies}

No appreciable change in physical characteristics, the results concluded that fast dissolving tablets of salbutamol sulphate were stable during accelerated stability conditions up to three months. (Data not shown).

\section{CONCLUSION}

Finally it is concluded that disintegrating properties of the mucilage and husk powder of Plantago ovata has been studied in comparison with croscarmellose sodium and

\section{REFERENCES}

1. Augsburger L, Hoag S. Tablets: Orally Disintegrating Tablets and Related Tablet Formulations. Pharmaceutical Dosage Forms $3^{\text {rd }}$ Edn, Vol 2, Rational Design and Formulation; Informa healthcare USA, Inc; 2008. P.293-294.

2. Mahaveer PR, Orally disintegrating tablets: A future prospectus, Int J Pharm Sci Bi, 1998, 3(1),71-79.

3. Bhise SD, Isolation and Evaluation of Natural Superdisintegrant from Plantago ovata in MDTs of Telmisartan, IJPI's Journal of Pharmaceutics and Cosmetology, 2012, (2), 10-21.

4. Raghavendra Rao NG, Formulation and evaluation of fast dissolving tablets of Metoprolol tartrate using natural superdisintegrant, Int J Pharm Clin Res, 2010, 2 (1), 40-45.

5. Sharma V, Comparison of various natural superdisintegrants in the formulation of fast dissolving Carvedilol tablet, sodium starch glycolate. The isolated natural disintegrant exhibited faster drug dissolution in comparison to the synthetic superdisintegrants. These formulations improve the bioavailability and effective therapy using Plantago ovata mucilage as natural superdisintegrant. Therefore, in the years to come, there will be continued interest in natural mucilages and their modifications aimed at the development of better materials for drug delivery systems.

\section{ACKNOWLEDGMENTS}

The authors are grateful to Glenmark Pharmaceuticals Ltd. Nashik and Gayatri Psyllium husk powder, Unjha Gujarat for providing gift samples of Salbutamol sulphate and Plantago ovata husk powder also thankful to Department of Quality Assurance Techniques, R. G. Sapkal College of Pharmacy, Anjaneri, Nashik for providing the required facilities.

International Journal of Pharmaceutical Sciences and Research, 2012, 3(10), 3947-3954.

6. Fischer MH, Nanxiong Yu, Gary R. The Gel-forming Polysaccharide of Psyllium Husk (Plantago ovata Forsk), Carbohydrate Research, 2004 339, 2009-2017.

7. British Pharmacopoeia 1988, Published by B.P. Commission Office, Vol-I, P.321-322.

8. The Indian Pharmacopeia 1996, Government of India, Ministry of Health and Family Welfare, Vol-III, Published by The Indian Pharmacopeia Commission, Ghaziabad, P.416.

9. USP-NF 2008, The Official Compendia of Standards, Asian Edn, Vol- 1, P.266-267,368, 675.

10. ICH guidelines. Stability testing of new drug substances and products. Q1A (R2),2003. 